\title{
Essential medical laboratory services: their role in delivering equitable health care in Malawi
}

\author{
RJ Dacombe', SB Squire², ARC Ramsay ${ }^{3}$, HT Banda ${ }^{4}$, I Bates ${ }^{2}$
}

\author{
' Liverpool Associates in Tropical Health \\ ${ }^{2}$ Liverpool School of Tropical Medicine, UK \\ ${ }^{3}$ Tropical Disease Research, World Health Organisation \\ ${ }^{4}$ REACH Trust, Lilongwe, Malawi
}

\begin{abstract}
This paper examines the establishment of Essential Medical Laboratory Services (EMLS) and their crucial role for delivering equitable health care to the poor population of Malawi as part of the Essential Health Package. We examine each of the major areas identified for intervention
\end{abstract}

(maternal health, malaria, tuberculosis and HIV) and how the EMLS has addressed these issues. We also discuss the potential for and current limitations of extending these services closer to the community.

\section{Introduction}

Sixty-five percent of Malawi's approximately 12 million people are defined as poor1. The major infections (malaria, HIV and tuberculosis) and the worsening maternal and infant mortality rate have a disproportionate effect on the poor. The poor may be denied access to health provision due to charges, distance and poor logistical support of services. Individuals may be discouraged from seekin medical care due to misdiagnosis, delays in receiving treatment and interaction with health providers ${ }^{2,3}$. This is clearly an inequitable situation with the majority of the population being unable to access effective health services. Inadequate public health care has a greater effect on poor fami${ }^{1}$ ies as they lack any savings with which to cover the costs of i11ness. Those suffering from disease will suffer increased financial burden due to an inability to work as well as the more direct costs such as transport and costs of treatment ${ }^{4,5}$.

An effective laboratory service is an essential part of a functional health service. Laboratories provide confirmatory diagnosis and improved management of disease, essential public health information and disease surveillance. Due to this wide-ranging role, laboratories are an important part of many disease control programmes; yet laboratory services are often ignored or taken for granted. This means that laboratory services have been under-resourced, poorly managed and rendered ineffective in many developing countries. Laboratories require a good logistical supply and utility services to be in place to function properly, something which is difficult to achieve even in urban population centres in Malawi. This makes the provision of laboratory services even more problematic and difficult to sustain in remote rural areas. In Malawi, along with many other developing countries, the greatest limiting factor on laboratory capacity is the availability of laboratory personne $1^{6,7,8}$.

Laboratory services should be developed to address the greatest needs within the community as a whole, and to allow them to operate within the above constraints. Ideally they should be part of an integrated health package to allow resources to be shared effectively. Previously in Malawi, disease programmes tended to be organised independently, thus affecting the integrated growth of the laboratory service. As part of the national poverty reduction strategy the Ministry of Health and Population developed the Essential Health Package to improve access to essential health care for the most common diseases affecting the poor. The essential medical laboratory services (EMLS) project was set up to establish evidence-based recommendations for the improvement of the district laboratory service to support the essential health package ${ }^{9}$. The establishment of the Sector Wide Approach (SWAp) in Malawi should help the integration of laboratory services and health services as a whole; though the risk of certain areas attracting disproportionate shares of funds at the expense of others remains ${ }^{10}$.

\section{Safe Motherhood}

The establishment of safe motherhood services is seen as a priority in Malawi, with maternal mortality estimated at 1,800 per 100,000 live births in 2004. Services are tiered into basic emergency obstetric care (BEmOC) and comprehensive emergency obstetric care (CEmOC), both of which require elements of laboratory support. As a way of promoting safe motherhood and better outcomes for their babies, antenatal screening services for anaemia, HIV, syphilis and malaria are pivotal.

The EMLS project piloted the use of the rapid plasma reagin (RPR) test for antenatal syphilis testing. The project found that it was feasible to use this test in primary health care antenatal clinics as it is relatively quick and requires little equipment.

As part of antenatal screening services, the WHO recommends that all pregnant women have a haemoglobin $(\mathrm{Hb})$ measurement taken at least once during their pregnancy ${ }^{1 !}$. Hb measurement is also important in assessing patients with malaria and may be a proxy indicator used in decisions about ARV treatment. The quality of routine $\mathrm{Hb}$ measurement in Malawi was highlighted by the EMLS project as a cause for concern and led to a recommendation to adopt a more accurate, reproducible method such as the HemoCue method ${ }^{12}$.

Due to the wide-ranging need for $\mathrm{Hb}$ estimation in health care, it would be advantageous if this service could be brought into the primary health care setting. The haemoglobin colour scale is a potential technology that is simple and quick. There is some evidence to suggest that this scale may be more effective for anaemia diagnosis than using clinical signs alone, which is the current method for diagnosing anaemia in primary care facilities. The scale is less expensive than other methods for estimating haemoglobin and is suitable for large-scale use at primary health care level ${ }^{12}$. The main concern about introducing Hb estimation at the community level is the scale of the problem. The EMLS project estimated the number of health care attendees suffering from severe anaemia to be 1 in 12 , a number with the potential to overwhelm the secondary health care service. This indicates the need to carefully evaluate the potential effects of introducing laboratory tests in primary care facilities on the upstream referral system.

Blood transfusion is an important requirement for BemOC, with a third of direct maternal deaths resulting from haemorrhage. An effective blood transfusion service is also vital for many activities in the health service - from major surgery to treatment of severe anaemia in children. Screening blood for infection was identified as the most costly processes amongst the critical laboratory services ( $59 \%$ of total costs) ${ }^{9}$, but an effective screening system is essential to reduce the risks of transfusion-related infections due to the high prevalence of blood-borne disease in Malawi ${ }^{13}$. Malawi is gradually introducing a national blood 
transfusion service. In districts not yet served by this national system, blood continues to be provided by patients' relatives and screened by the district laboratory. This is an additional burden on households of the sick, who bear the costs of transporting these relatives to a district hospital.

\section{Malaria}

Malaria is one of major disease problems for Malawi, having a higher morbidity and mortality amongst pregnant women and young children. It is associated with poverty as the poor have difficulty in accessing diagnosis, treatment and preventative measures. Presently the majority of malaria diagnosis at primary and secondary level facilities is based on symptoms and treated empirically with sulphadoxine pyrimethamine (SP). This approach leads to a significant amount of misdiagnosis ${ }^{2}$ and may promote development of resistance to SP, which could result in an urgent need to change the treatment of malaria. This treatment would almost certainly involve more expensive and toxic treatments requiring a more specific malaria diagnosis.

Light microscopy is the "gold-standard" method for detection of malaria parasites in blood films but is difficult to provide in peripheral health clinics. This is due to the high investment in technical expertise, quality monitoring, microscope maintenance and time needed to correctly identify malaria parasites in blood films ${ }^{14}$. However, malaria rapid diagnostic devices may offer a solution to bringing an effective diagnosis of malaria to primary health care ${ }^{15}$. These devices are quick and simple, using immunochromographic tests to detect malaria-specific antigens. However these devices have limitations in sensitivity at increased temperatures and specify for plasmodia other than Plasmodium faciparum. As with any test, quality assurance systems have to be established for these to monitor the performance of the technology and the operator; the lack of skilled laboratory supervisors are likely to be a limiting factor in making these tests available at the primary care level.

\section{Tuberculosis}

Tuberculosis is another disease where microscopy is the standard method of diagnosis. The current international recommendation is that all patients (with a few specified exceptions) have sputum specimens examined for TB bacilli before TB treatment is considered (see Malawi DOTS strategy and Malawi NTP Control Manual). Therefore sputum smear microscopy has been the gateway to TB treatment for most patients in Malawi. As reliable rapid diagnostic tests for tuberculosis (equivalent to HIV testing or rapid malaria diagnostic tests) have not yet reached the market-place, sputum smear microscopy is set to remain a rate limiting step for poor patients attempting to access TB services ${ }^{16,17}$. The key issues facing delivery of equitable sputum smear diagnostic services for tuberculosis are:

The number of specimens required to inform decision-making about treatment. In addition to the conventional considerations of sensitivity and specificity, there are also related considerations of laboratory workload and speed of delivery of results to patients.

The speed and reliability of transport of specimens from the local area to microscopy centres.

Quality assurance of smear microscopy. This includes internal quality control and linkage to External quality assurance mechanisms.

The distribution and availability of microscopy centres in relation to population density and burden of TB disease.

Taking each of these issues in turn, it is clear that recent work in Malawi through the EMLS project and the EQUI-TB Knowledge programme provide useful pointers for the way forward in promoting the development of equitable diagnostic services for TB.

Malawi Medical Journal: Special Edition on Equity II
The current international recommendation is that each TB suspect should produce three consecutive sputum specimens before TB disease categorisation can be achieved. There is evidence, however, that patients drop out of the associated diagnostic process because it requires repeated visits to diagnostic centres $^{18,19}$. Early results from operational research suggest that it may be possible to consider relying on fewer specimens in the diagnostic process in order to maximise the number of smear positive patients starting treatment ${ }^{20}$ with the additional advantage of reducing laboratory workload ${ }^{21}$.

The issue of chemical digestion of sputa in improving the sensitivity of smear microscopy in low income settings is a topic of much debate and may have the potential to reduce the number of specimens required.. However, the most realistic choice to aid the expansion of TB microscopy closer to the community is the bleach sedimentation method ${ }^{23}$. Bleach is a cheap and widely available chemical and therefore highly suited to resourcepoor areas. Sedimentation requires no additional equipment to perform; unlike the other widely studied methodologies using centrifugation Although the bleach sedimentation method may not significantly increase the sensitivity of TB microscopy it does have an important intrinsic advantage of disinfection; reducing the risk of infection once the sample has been processed. Microscopists have also commented that addition of bleach renders the smears easier to read. The obvious disadvantage of this method is it renders the sample unsuitable for culture, but this is rarely an issue in areas where microscopy is the only diagnostic technique used ${ }^{24}$.

In addition to acting as a barrier to patients accessing services, time delays in transporting sputum specimens may also adversely affect the sensitivity of tuberculosis microscopy. A study in Malawi by Banda et al..$^{25}$ examined the effect of prolonged storage on acid-fastness using the ZN stain and found no loss of positivity after 8 weeks, though 3 samples did dry out. This is encouraging for a laboratory system with a limited number of microscopy centres, as sputa may be produced at the health post and then transported with no loss of sensitivity. It is worth noting however that the ambient temperatures in this experiment did not exceed $25 \circ \mathrm{C}$; far lower than highest seasonal temperatures in Malawi, so further work including those temperatures is needed.

The EMLS programme demonstrated that it is possible to promote internal quality assurance of TB smear microscopy at the district level in Malawi ${ }^{26}$. It is important to include the processes of transportation and slide preparation in the monitoring. Through the national tuberculosis programme, this EMLS work has fed into the development of a network of external quality control checks for district laboratories using a blinding rechecking method. Additionally, the central reference laboratory (CRL) is being strengthened to allow drug resistance monitoring to be performed. This is currently being evaluated by the CRL, linking to an international external quality assurance scheme.

\section{篔篦}

HIV diagnosis is increasingly available in Malawi close to the community due to the availability of affordable, rapid, point-ofcare diagnostic tests. Rapid testing for HIV has been subject to a large degree of evaluation and development to allow for the selection of robust tests suitable for field conditions common to developing countries. As with rapid malaria diagnostic tests, a good system of quality assurance must be in place to ensure accurate results. These HIV tests are limited by an inability to diagnose HIV infection in the very young and those very recently infected. Diagnosis of HIV in these groups requires specialist testing not suitable for primary health care.

Laboratories need to play a key role in the provision of anti- 
retroviral (ARV) therapy for HIV infection. The usual immunological indicator used in Western countries to assess a patient's need for therapy is CD4 counting and HIV RNA load. These carry a significant cost for equipment and reagents, specialised maintenance and are generally unsuitable for settings with poor power supply. Malawi, and countries have developed policies that use clinical staging to assess those suitable for treatment. This approach may be more equitable than relying on laboratory tests because CD4 counts are not widely available. Other common laboratory tests may be suitable for assessing a patient's need to access ARVs. Both $\mathrm{Hb}$ and total lymphocyte count have been examined for use in combination with clinical staging. With the high level of anaemia within poorer communities, $\mathrm{Hb}$ may not be a sensitive enough tool to help define a treatment threshold. Total lymphocyte count appears to correlate well with the risk of disease progression both in children ${ }^{28}$ and adults ${ }^{29}$. This approach may be a suitable compromise between a full, high cost laboratory dependant programme and simple clinical staging, but these proxy indicators need evaluating for feasibility and effectiveness in 'real-life' settings.

\section{Conclusion}

The laboratory has a critical role to play in supporting the delivery of Malawi's Essential Health Package, but the resource requirements of a laboratory service and a lack of understanding of how they function can be barriers to allocating adequate support. However without an effective laboratory service patient diagnosis and care is often compromised, expensive drug treatments are squandered, diagnoses are missed and information about public health is inaccurate. It is also impossible to measure the true effectiveness of interventions and to conduct accurate disease surveillance. Malawi provides an example to other developing countries about how to collect locally relevant evidence about the priorities for laboratory services and to implement these findings to support the delivery of an essential health package. Malawi has taken a pragmatic approach to the provision of laboratory services in the public sector by identifying and addressing, as simply as possible, the most serious health problems. This progress must continue to be supported to improve the equitable delivery of health care in Malawi and to be able to identify and meet the health challenges of the future.

\section{References}

1. National Economic Council. Profile of poverty in Malawi 1998: An Analysis Of Malawi Integrated Household Survey 1997 1998. Poverty Monitoring System. Zomba: National Statistics Office; 2004.

2. Amexo M, Tolhurst R, Barnish G, Bates I. Malaria misdiagnosis: effects on the poor and vulnerable. Lancet 2004;364:1896-98.

3. Petti CA, Polage CR, Quinn TC, Ronald AR, Sande MA. Laboratory medicine in Africa: a barrier to effective health care. Clin Infect Dis. 2006;42:377-82.

4. Nhlema Simwaka B, et al, The Malawi National Tuberculosis Programme: an equity analysis. Malawi Med J 2006;18(1):

5. Makwiza I, et al. Who has access to counselling and testing and anti-retroviral therapy in Malawi? An equity analysis. Malawi Med J 2006; 18(1):

6. Ministry of Health, Liverpool School of Tropical Medicine. Essential Medical Laboratory Services Report. 1998-2002. Lilongwe, Liverpool: Government of Malawi; 2002.

7. Ministry of Health. Human resources in the health sector: towards a solution (draft). Lilongwe: Ministry of Health; April 2004.

8. Harries AD, Zachariah R, Bergstrom K, Blanc L, Salaniponi FM, EIzinga G. Human resources for control of tuberculosis and HIVassociated tuberculosis. Int J Tuberc Lung Dis. 2005;9(2):128137.

9. Mundy CJF, Bates I, Nkhoma W, Floyd K, Kadewele G, Ngwira M, Khuwi A, Squire SB, Gilks CF. The operation, quality and cost of a district hospital laboratory service in Malawi. Trans $\mathrm{R}$ Soc Trop Med Hyg. 2003;97:403-408.

10. Bates I. Report on progress of scaling up Essential Medical Laboratory Services within the context of the health SWAp and new 'Treatment of AIDS' policy. 2005.

11. World Health Organisation. Care of mother and baby at the health centre: a practical guide. Introduction and table 2.3; Prenatal care. WHO/MSM/94.2-Rev 2; Geneva: WHO; 1994.

12. Medina Lara A, Mundy C, Kandulu J, Chisuwo L, Bates I. Evaluation and costs of different haemoglobin methods for use in district hospitals in Malawi. J Clin Pathol. 2005;58:56-60.

13. Candontti D, Mundy C, Kadewele G, Nkhoma W, Bates I, Allain JP. Serological and Molecular Screening for Viruses in Blood Donors From Ntcheu, Malawi: High Prevalence of HIV-1 Subtype C and of Markers of Hepatitis B and C viruses. J Med Virol. $2001 ; 65: 1-5$.

14. Mundy C, Ngwira M, Kadewele G, Bates I, Squire SB, Gilks CF. Evaluation of microscope condition in Malawi. Trans R Soc Trop Med Hyg 2000;94:583-584.

15. Moody A. Rapid Diagnostic Tests for Malaria Parasites. Clin Micro Rev. 2002;15(1):66-78.

16. Hargreaves NJ, Kadzakumanja O, Phiri S, Nyangulu DS, Salaniponi FM, Harries AD, Squire SB. What causes smearnegative pulmonary tuberculosis in Malawi, an area of high HIV seroprevalence? Int J Tuberc Lung Dis. Feb 2001;5:113-22.

17. Squire SB, Belaye A K, Kashoti A, Salaniponi FML, Mundy CJF, Theobald S, Kemp J. "Lost" smear positive pulmonary tuberculosis cases; where are they and why did we lose them? Int $J$ Tuberc Lung Dis 2005;9:25-31.

18. Kemp J, Squire SB, Nyasulu K, Salaniponi FML. Is tuberculosis diagnosis a barrier to care? Trans R Soc Trop Med 1996; 90:472.

19. Squire SB, Nyasulu IK, Kanyerere H, Salaniponi FML. Why bother with three sputum smears for case-finding in tuberculosis control? Trans R Soc Trop Med 1996;90:478.

20. Squire SB, Gondwe M, Michongwe J, Salaniponi FML, Mundy C, Kemp J. The feasibility of using a single "on-spot" sputum smear for TB case finding. Int $\mathbf{J}$ Tuberc Lung Dis 2001; 5: S172.

21. Harries AD, Mphasa NB, Mundy C, Banerjee A, Kwanjana JH, Salaniponi FML. Screening tuberculosis suspects using two sputum smears. Int J Tuberc Lung Dis. 2000; 4: 36-40.

22. Lawson L, Yassin MA, Ramsay A, Olajide I, Thacher TD, Davies PDO, Squire SB, Cuevas LE. Microbiological validation of smear microscopy after sputum digestion with bleach; a step closer to one-stop diagnosis of pulmonary tuberculosis.

Tuberculosis. 2006; 86:34-40.

23. Yassin MA, Cuevas LE, Gebrexabher H, Squire SB. Efficacy and safety of short-term bleach digestion of sputum in casefinding for puimonary tuberculosis in Ethiopia. Int J Tuberc Lung Dis 2003;7: 678-683.

24. Ramsay A, Squire SB, Siddiqi K, Cunningham J, Perkins M. The bleach microscopy method and case detection for TB control. Int J Tuberc Lung Dis 2006; 10: 1-3.

25. Banda HT, Harries AD, Boeree MJ, Nyirenda TE, Banerjee A, Salaniponi FML. Viability of stored sputum specimens for smear microscopy and culture. Int J Tuberc Lung Dis 2000; 4: 272-274.

26. Mundy CJF, Harries AD, Banerjee A, Salaniponi FM, Gilks CF, Squire SB. Quality assessment of sputum transportation, smear preparation and AFB microscopy in a rural district in Malawi. Int J Tuberc Lung Dis 2002; 6: 47-54.

27. Kemp J, Boxshall M, Nhlema B, Salaniponi FML, Squire SB. Application of a Geographical Information System (GIS) to examine the relationship between socio-economic status and access to care for TB in urban Lilongwe: Int $J$ Tuberc Lung Dis. 2001;5: Supplement 1,S167.

28. HIV Paediatric Prognostic Markers Collaborative Study. Use of total lymphocyte count for informing when to start antiretroviral therapy in HIV-infected children: a meta-analysis of longitudinal data. Lancet. 2005;366:1868-74.

29. Mekonnen Y, Dukers NHTM, Sanders E, Dorigo W, Wolday D, Schaap A, Geskus RB, Coutinho RA, Fontanet A. Simple markers for initiating antiretroviral therapy among $H I V$-infected Ethiopians. AIDS. 2003;17:815-819. 